\title{
0IS0, traitement informatisé de la prise en charge en oncogénétique clinique
}

Céline Guien ${ }^{1,2}$, Aurélie Fabre ${ }^{3,4}$, Arnaud Lagarde ${ }^{3,4}$, David Salgado ${ }^{1}$, Catherine Gensollen-Thiriez ${ }^{3}$, Hélène Zattara $^{3}$, Christophe Beroud ${ }^{1,3}$, Sylviane Olschwang ${ }^{1,2,3}$

Mots clés

Oncogénétique

Diagnostic

Dépistage

Traitement automatisé

Réseau régional
1. Aix-Marseille université, Inserm, GMGF UMR S 910, 27, boulevard Jean-Moulin, 13385 Marseille, France

2. RGDS, hôpital Clairval, 317, boulevard du Redon, 13009 Marseille, France

3. AP-HM Timone, département de génétique médicale, 264, rue Saint-Pierre, 13005 Marseille, France

Correspondance :

Sylviane Olschwang, Faculté de médecine, unité Inserm, UMR_S910, 27, boulevard Jean-Moulin, 13005 Marseille, France.

sylviane.olschwang@inserm.fr

\section{Résumé}

Introduction > L'oncogénétique est une démarche au long cours, qui exige une relation de confiance entre le consultant et l'ensemble du personnel soignant, et suppose une communication intrafamiliale afin que chacun puisse bénéficier d'une prise en charge optimale. Elle nécessite le recueil de nombreux documents et des interactions fréquentes entre les différents acteurs. Nous présentons ici la création d'un outil qui tient compte des exigences de cette activité.

Méthodes > L'outil a été développé selon une approche modèle-vue-contrôleur avec le système relationnel PostgreSQL 9.3. Le site web a été développé avec les langages PHP 5.3, HTML5 et CSS3, ainsi que des fonctions en JavaScript et jQuery-AJAX. Deux modules ont également été utilisés, FPDF et PHPMailer.

Résultats > L'outil permet l'interaction d'intervenants multiples, la gestion des données cliniques, l'enregistrement et le classement des documents, l'édition de comptes rendus et de courriers, l'envoi de courriers électroniques, la gestion des calendriers et des rendez-vous. Une panoplie de requêtes a été élaborée afin d'automatiser la veille des dossiers sans perte d'information, de réaliser du publipostage et d'évaluer l'activité.

Discussion > L'outil a été construit spécifiquement pour les consultations et le suivi des personnes en oncogénétique et comporte tous les éléments nécessaires et adaptés à cette activité. II bénéficie d'une autorisation de la CNIL, est accessible par Internet, et cet accès est restreint et sécurisé. Il est doté d'un guide de remplissage en ligne. Développé par une structure académique, il est souple et évolutif, et permet de répondre aux besoins de l'oncogénétique.

\footnotetext{
4 ont également contribué.
} 
Keywords

Oncogenetics

Diagnosis

Follow-up

Automated management

Regional network

\section{Summary}

OISO, automatic treatment of patients management in oncogenetics

Introduction > Oncogenetics is a long-term process, which requires a close relation between patients and medical teams, good familial links allowing lifetime follow-up. Numerous documents are exchanged in between the medical team, which has to frequently interact. We present here a new tool that has been conceived specifically for this management.

Methods > The tool has been developed according to a model-view-controler approach with the relational system PostgreSQL 9.3. The web site used PHP 5.3, HTML5 and CSS3 languages, completed with Javascript and jQuery-AJAX functions and two additional modules, FPDF and PHPMailer.

Results > The tool allows multiple interactions, clinical data management, mailing and emailing, follow-up plannings. Requests are able to follow all patients and planning automatically, to send information to a large number of patients or physicians, and to report activity.

Discussion > The tool has been designed for oncogenetics and adapted to its different aspects. The CNIL delivered an authorization for use. Secured web access allows the management at a regional level. Its simple concept makes it evolutive according to the constant updates of genetic and clinical management of patients.

\section{Introduction}

Dans les années 1990, la découverte de gènes de susceptibilité au cancer a permis à une pratique médicale nouvelle de voir le jour, la génétique oncologique ou oncogénétique. Elle a pour objet d'identifier les personnes à risque élevé de cancer et de proposer une prise en charge adaptée à ce risque. Chez les personnes porteuses d'une altération d'un gène de prédisposition majeure, à transmission autosomique dominante dans la plupart des cas, l'anomalie génétique peut être à l'origine du développement d'un ou de plusieurs cancers. Dans certains cas, l'altération de plusieurs gènes de prédisposition mineure est nécessaire pour observer une manifestation clinique. Aux facteurs génétiques transmissibles s'ajoutent les altérations génétiques acquises dans les cellules somatiques, et qui provoquent leur transformation maligne. Certains de ces mécanismes somatiques sont en outre sous la dépendance de facteurs environnementaux.

La génétique oncologique se distingue sur plusieurs plans de la génétique médicale classique.

Une prédisposition génétique au cancer peut être évoquée sur la reconnaissance de certaines particularités chez une personne, et très souvent dans la même branche familiale. Ces particularités concernent les organes touchés, l'âge d'apparition des lésions, leur nombre, leurs caractéristiques histologiques, le lien de parenté des différentes personnes atteintes d'une famille... Une expertise soigneuse doit donc être faite avant d'entreprendre l'analyse moléculaire qui permettra de compléter I'hypothèse clinique [1-3].

L'identification de la prédisposition permet de fournir une information non ambiguë à une personne qui demande un conseil génétique, et de proposer aux personnes ayant déjà été atteintes d'un cancer comme à leurs apparentés une prise en charge adaptée à leur risque, dont le but est de dépister suffisamment tôt les cancers pour les guérir, voire de les prévenir par des interventions chirurgicales prophylactiques. Cette étape nécessite d'avoir au préalable identifié la mutation responsable de la pathologie familiale. Ceci implique qu'une personne ayant une probabilité non négligeable d'être porteuse d'une altération sur un gène de prédisposition accepte d'être orientée vers une consultation de génétique puis consente à une analyse moléculaire [4-6].

\section{Déroulement des consultations}

C'est une démarche en plusieurs temps, initiée par les unités de consultation pluridisciplinaire qui recueillent les données anamnestiques individuelles et familiales, et proposent éventuellement une consultation d'oncogénétique.

En effet, il n'existe aucun signe pathognomonique permettant, à partir de la seule présentation clinique d'un cancer, d'identifier une prédisposition héréditaire et le gène responsable, mais un faisceau d'arguments permet généralement d'émettre une hypothèse diagnostique :

- les arguments cliniques : diagnostic à un âge inhabituel, existence de cancers primitifs multiples, histoire familiale de cancers ;

- les arguments bio-pathologiques : caractéristiques histologiques, immunohistochimiques ou biologiques des cellules tumorales, attestées par des comptes rendus.

L'indication d'analyse moléculaire repose sur le calcul de la probabilité de l'existence d'une mutation constitutionnelle délétère, responsable d'une prédisposition héréditaire au cancer. Le choix du seuil, à partir duquel l'analyse est proposée, varie en 
fonction de l'hypothèse diagnostique, et résulte d'un compromis entre sensibilité et spécificité, qui définissent l'efficacité de la prise en charge. Une organisation locale des consultations autour d'un réseau de médecins spécialistes qui participent à la définition et à l'analyse des indications, est une solution souple et évolutive permettant une bonne efficacité.

\section{La consultation initiale}

La consultation initiale est une consultation longue, au cours de laquelle le recueil des informations familiales est focalisé sur les cancers dont ont éventuellement été atteintes les générations proches. Lorsque l'évaluation à cette première étape confirme l'existence d'un risque élevé, un examen moléculaire des caractéristiques génétiques est proposé. Il ne peut être entrepris qu'après avoir fourni à la personne concernée l'ensemble des informations relatives aux caractéristiques de la maladie recherchée, aux moyens de la détecter, aux possibilités de prévention et de traitement, et avoir recueilli son libre consentement par écrit avec signature. La réalisation de cet examen est réglementée [7]. À cette étape, le dossier médical d'oncogénétique s'étoffe de divers documents : les comptes rendus médicaux relatifs à la maladie tumorale du consultant, les comptes rendus d'analyses complémentaires (histologie, génétique somatique) et, le cas échéant, l'arbre généalogique, le consentement à l'analyse génétique constitutionnelle, la prescription de cette analyse.

\section{Consultation(s) intermédiaire(s)}

Des informations complémentaires sollicitées au cours de la consultation initiale peuvent être recueillies lors d'une consultation intermédiaire par le généticien et/ou le consultant (résultats histologiques, immunohistochimiques, anatomopathologiques, etc.). Ces informations peuvent conduire à la proposition d'une analyse génétique qui n'aurait pas été proposée lors de la consultation initiale ou, si une analyse n'est pas retenue, à la proposition d'une prise en charge adaptée en fonction du contexte personnel et familial. La consultation intermédiaire peut également concerner les personnes qui souhaitent un délai de réflexion avant de consentir à l'analyse.

\section{La consultation d'annonce}

À réception du compte rendu d'analyse génétique, le généticien adresse une convocation écrite au consultant lui proposant une consultation d'annonce. La restitution du résultat de l'examen des caractéristiques génétiques est également réglementée [7]. La consultation d'annonce est une consultation longue et a pour but de proposer une prise en charge adaptée au risque estimé. L'identification d'une anomalie génétique constitutionnelle nécessite sa confirmation sur un prélèvement de contrôle, ainsi que la présentation des recommandations (dépistage, chirurgie prophylactique) en accord avec les référentiels nationaux, qui peuvent être adaptés à l'histoire personnelle et familiale. Un examen génétique prédictif peut être proposé aux personnes apparentées, et il est expliqué à cette étape que la diffusion de l'information à la parentèle est une obligation légale [8]. À l'issue de la consultation, une inclusion dans le réseau régional de suivi des personnes présentant une prédisposition à certains cancers peut être proposée. Ce réseau assure le respect et la mise à jour du calendrier des examens de dépistage, leur réalisation dans les conditions de qualité requises, le recueil des résultats, et l'adaptation de la prise en charge en fonction des résultats et de l'évolution des référentiels nationaux. Le dossier médical est donc à ce stade complété par le compte rendu d'analyse génétique, l'attestation de délivrance du résultat et le cas échéant, le consentement à l'inclusion dans le réseau de suivi régional.

En cas de résultat négatif, c'est-à-dire lorsque aucune variation génétique pathogène n'est identifiée, la probabilité résiduelle que le consultant soit porteur d'une mutation constitutionnelle non identifiable doit être réévaluée afin de proposer une prise en charge adaptée au risque. Si elle est jugée élevée, une prise en charge de type haut risque génétique est discutée lors d'une réunion de concertation multidisciplinaire d'oncogénétique. Si cette prise en charge est validée, elle suppose une nouvelle consultation et une possible inclusion du consultant dans le réseau régional de suivi. Le compte rendu de réunion de concertation multidisciplinaire est joint au dossier.

\section{Les consultations de suivi}

Il est ensuite utile de proposer des consultations de suivi aux personnes dont le risque de cancer est élevé, sur l'existence d'une mutation délétère ou la base d'arguments cliniques personnels et familiaux. Ces consultations peuvent être physiques ou consister en des échanges téléphoniques ou écrits en fonction de la situation et des souhaits de chaque consultant. Le réseau de suivi propose et adapte la prise en charge en fonction des résultats des examens de dépistage, de la survenue éventuelle d'interventions chirurgicales (prophylactiques ou curatives) et de l'évolution des recommandations. Il est en étroite relation avec les réunions de concertation multidisciplinaire d'oncogénétique, auxquelles il réfère les dossiers susceptibles de requérir un changement important de prise en charge. Les comptes rendus des interventions médicales et chirurgicales et des examens de dépistage sont recueillis au cours de ces consultations. Le réseau de suivi est également en lien avec les équipes médicales assurant la prise en charge du patient afin de les informer de l'inclusion du consultant, de lui exposer les recommandations personnalisées et de recueillir les comptes rendus afférents au suivi.

Le soutien de l'Institut national du cancer a permis la création de programmes régionaux de prise en charge des personnes à risque très élevé de cancers qui doivent s'appuyer sur des logiciels d'aide au suivi afin de satisfaire au cahier des charges (enregistrements, procédure de rappel des rendez-vous, recueil des résultats des examens de suivi, messages d'alertes, etc.). 
Actuellement, plusieurs solutions commerciales ont été développées ou adaptées au moyen d'investissements financiers élevés [9].

Nous présentons ici la création de novo d'un outil académique qui tient compte des exigences de cette activité, permettant l'interaction d'intervenants multiples, la gestion des données cliniques, l'enregistrement et le classement des documents, l'édition de comptes rendus et de courriers, l'envoi de courriers électroniques, la gestion des calendriers et des rendez-vous. Une panoplie de requêtes plus ou moins complexes a également été développée afin d'automatiser la veille des dossiers sans perte d'information, de réaliser du publipostage et d'évaluer facilement l'activité.

\section{Matériel et méthodes}

\section{Matériel}

Les éléments constitutifs du dossier médical des patients concernent des informations administratives relatives aux patients et aux équipes médicales qui les prennent en charge, des données médicales, biologiques et génétiques relatives aux patients et aux personnes qui leur sont apparentées. Le dossier contient également des documents relatifs à la pathologie tumorale des patients et de leurs proches (comptes rendus opératoires, histologiques), à la prise en charge oncogénétique initiale (comptes rendus d'analyse de génétique somatique, constitutionnelle, consentements, attestations) et au suivi des patients (modalités et calendrier de suivi, comptes rendus d'examens de dépistage ou opératoires).

\section{Méthodes}

La base de données a été développée avec le système de gestion de bases de données relationnelles objet PostgreSQL 9.3 [10]. Le site web associé a été développé avec les langages PHP 5.3 [11], qui permettent l'écriture de scripts généralistes, spécialement conçus pour le développement d'applications web, HTML5 [12] et CSS3 [13], qui donnent le style aux pages web.

Des fonctions en JavaScript [14] et jQuery-AJAX [15] ont été utilisées dans les pages web afin de rendre l'affichage et le traitement des données dynamiques, de modifier le contenu des pages sans avoir à les recharger, et de produire des analyses statistiques.

Deux modules développés en PHP ont également été utilisés. Le premier, FPDF [16], permet de réaliser des PDF. Le second, PHPMailer [17], permet d'envoyer des messages électroniques.

\section{Résultats}

\section{Structure de la base de données}

L'architecture du code PHP suit un modèle Modèle-Vue-Contrôleur (MVC), qui sépare les différents composants du site web, affichage des informations, actions de l'utilisateur et accès aux données (figure 1). Le « Modèle » permet de gérer les données :

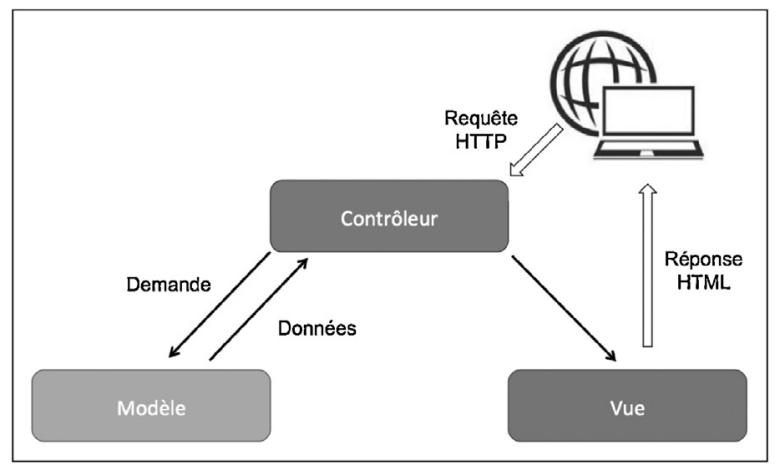

FIGURE 1

Schéma MVC

Le schéma modèle-vue-contrôleur traduit les interactions entre les différents modules depuis une requête formulée par l'utilisateur. Elles peuvent être à double sens (flèches doubles) ou à sens unique. Le modèle a la capacité de répondre à une question et matérialise visuellement la réponse sous forme de liste, qui peut ensuite être triée selon le choix de l'utilisateur.

ajout, modification, suppression et obtention des données. Il est constitué de classes PHP qui collectent variables et fonctions associées. Chaque classe représente une table. La " Vue " affiche les données renvoyées par le "Modèle ». Elle ne les modifie pas, mais peut les traiter afin qu'elles soient affichées à l'écran. Elle contient principalement du HTML et une partie de PHP permettant le traitement des données. Le "Contrôleur » fait le lien entre le " Modèle » et la "Vue ». Il gère les interactions avec l'utilisateur en utilisant les données du « Modèle » et en les envoyant à la "Vue » pour affichage.

La base de données OISO (Outil d'identification et de suivi en oncogénétique) est constituée de 31 tables, 180 champs et 44 liens, organisés en 4 parties (figure 2).

La première partie contient les données concernant les patients, les acteurs initiaux de la prise en charge oncogénétique, et les établissements de santé. Les utilisateurs de la base de données sont rattachés à un (des) établissement(s) de santé en fonction de leur(s) lieu(x) de consultations. Les établissements de santé sont organisés en groupes selon la région géographique dans laquelle sera éventuellement organisé le suivi des patients. La seconde partie est dédiée aux consultations, avec date, motif, informations recueillies ou délivrées, proposition(s) de prise en charge.

La troisième partie correspond au suivi des personnes à risque élevé de cancer, avec définition de la filière adaptée aux risques, du calendrier des examens, de l'équipe de prise en charge et des résultats des interventions médicales ou chirurgicales.

La dernière partie contient les documents, en lien avec les différentes étapes du parcours oncogénétique.

\section{Fonctions de la base de données}

Le menu principal propose à l'ensemble des utilisateurs plusieurs choix relatifs aux patients et à leur suivi : créer un nouveau 


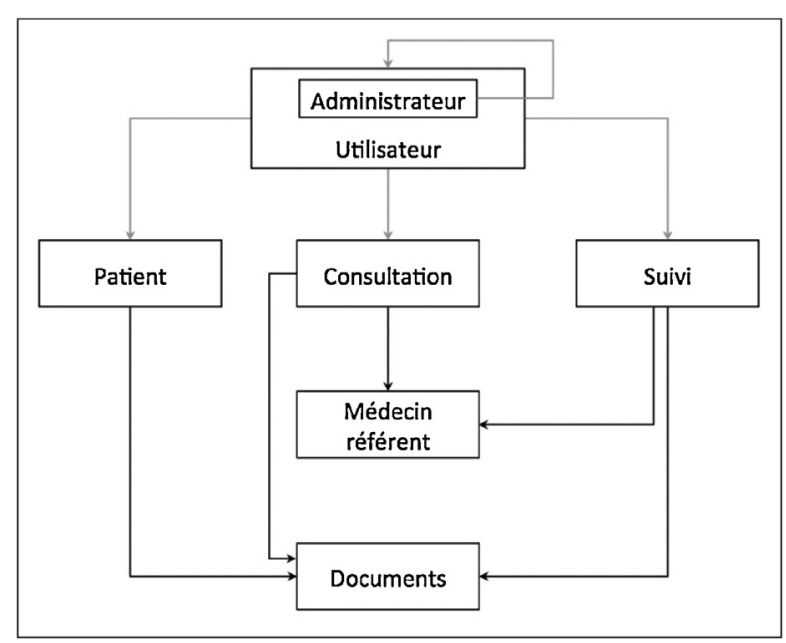

FIGURE 2

\section{Schéma conceptuel de la base de données}

Les utilisateurs, dont l'administrateur, ont différents droits d'action, de consultation et d'interrogation. Les flèches grises représentent les possibilités de création et modification par les utilisateurs. Les informations sont enregistrées dans des tables spécifiques, liées entre elles selon une architecture adaptée aux catégories d'informations. Les flèches noires représentent les liens entre les éléments en fonction de leur catégorie.

patient, lister les patients, les consultations, les patients ayant eu une prescription d'examen génétique pour lesquels le résultat n'est pas disponible, ceux informés de la disponibilité d'un résultat d'analyse génétique non venus pour la consultation d'annonce, les suivis en cours, les examens à prévoir dans les deux prochains mois, les examens programmés pour lesquels le compte rendu n'a pas été récupéré, les examens non programmés au-delà de la date prévue. Dans chaque liste, une requête ou un tri peut être fait sur chaque champ visible.

Un menu accessoire permet des requêtes sur l'ensemble des champs, une visualisation de l'activité des établissements, et un export des informations vers un tableur. L'accès à ce menu est restreint aux administrateurs.

Un second menu accessoire concerne l'administration de la base de données, à savoir la création et les contrôles d'accès des utilisateurs. Les utilisateurs ont la possibilité de changer leur mot de passe et d'enregistrer une signature numérique.

\section{Utilisation de la base de données}

Un utilisateur peut créer et modifier des formulaires de consultations et de suivi de ses patients, ajouter des documents. Seuls les administrateurs peuvent supprimer des formulaires. Chaque formulaire contient des données obligatoires. L'étape de validation d'un formulaire est associée à une vérification de la complétion et du format des champs obligatoires, avec message d'alerte si nécessaire. La plupart des items requis dans les champs obligatoires sont proposés sous forme de menu déroulant avec guide de remplissage. Les informations recueillies en consultation peuvent faire l'objet d'un compte rendu structuré. Cette option d'édition automatique est laissée au choix de l'utilisateur, avec possibilité de téléchargement au format pdf.

Lorsque la création d'un formulaire pour un nouveau patient est validée, l'ouverture d'un formulaire de consultation est automatiquement générée. De même, la prescription d'un suivi pour risque élevé génère l'ouverture d'un formulaire de suivi. Le remplissage de ces formulaires est optionnel. À réception d'un compte rendu d'analyse génétique, le résultat peut être saisi sur un nouveau formulaire de consultation qui permet d'inviter le patient par e-mail à prendre un rendez-vous de consultation. Un rappel peut être fait si nécessaire 4 mois après la première invitation. En cas de suivi pour risque élevé de cancer, plusieurs courriers standardisés sont également prévus, adressés au patient et/ou à chaque médecin assurant la prise en charge par courrier électronique ou papier (édition d'un format pdf). Ces courriers concernent l'inclusion dans le parcours de suivi, les informations relatives aux examens à programmer et le recueil des comptes rendus de ces examens.

\section{Discussion}

La prise en charge oncogénétique est une démarche complexe, qui met en relation des informations médicales individuelles et familiales concernant des maladies graves, et des informations concernant les caractéristiques génétiques constitutionnelles des personnes. C'est également une démarche longue pour les personnes dont le risque de développer un cancer est très élevé et qui se voient proposer un plan personnalisé de suivi au long cours. La structuration de cette activité grâce à un traitement informatisé des données est indispensable pour une prise en charge efficace, adaptée et évolutive des personnes. Les logiciels commerciaux sont très récents, peu nombreux, incomplets pour la plupart, et très onéreux [9]. L'activité d'oncogénétique s'étant déployée depuis une trentaine d'années, les praticiens ont développé des solutions locales souvent peu élaborées. La récupération des données issues de cette activité hétérogène est complexe et constitue un surcoût important lors de la mise en place d'une solution commerciale. De plus, une vigilance particulière doit être accordée aux conditions d'accès, afin de garantir la stricte confidentialité des données génétiques constitutionnelles. L'outil que nous proposons résulte d'une réflexion ancienne et de la transformation d'un outil précédemment développé sur un mode client-serveur au sein d'un seul établissement. Il a été construit spécifiquement pour les consultations et le suivi des personnes en oncogénétique et comporte ainsi tous les éléments nécessaires et adaptés à cette activité. II bénéficie d'une autorisation de la CNIL depuis 1998, et ses évolutions successives ont fait l'objet de plusieurs déclarations de modifications, quant à son hébergement et sa structure. Son accès est restreint et sécurisé. L'utilisateur se connecte via 
I'interface web en ligne avec son adresse e-mail, son mot de passe et un code d'accès attribué par e-mail à chaque demande de connexion. Après trente minutes d'inactivité, la connexion est interrompue. Les établissements dans lesquels il exerce sont définis par l'administrateur, de même que la région géographique dans laquelle ils se trouvent. L'utilisateur a uniquement accès aux informations des patients de ces établissements. L'ensemble des utilisateurs d'un établissement a accès aux informations des patients de cet établissement. Actuellement, les plans personnalisés de suivi sont disponibles pour la région Provence-Alpes-Côte d'Azur (réseau HerMION). L'extension de la structuration régionale nécessaire au suivi est en cours.

La saisie d'informations dans plusieurs champs a été rendue obligatoire, afin de garantir l'exhaustivité des recherches et l'exactitude des statistiques. Elle fait l'objet d'un guide de remplissage symbolisé par un "? " placé à côté des champs concernés.

Le recueil des informations relatives à l'histoire tumorale personnelle et familiale des consultants est souvent assorti de l'élaboration d'un arbre généalogique. Actuellement, l'outil ne permet pas la création intégrée des arbres, mais leur enregistrement depuis d'autres sources. L'ajout de cette option est en réflexion.

\section{Conclusion}

À l'ère du Dossier patient informatisé et du « zéro papier », ce logiciel dédié à l'oncogénétique comporte non seulement les éléments relatifs à tout type de consultation médicale (informations administratives, informations individuelles et familiales) mais également toutes les données inhérentes à cette spécialité. Il permet à partir d'un outil unique de générer automatiquement des comptes rendus de consultation qui peuvent être envoyés directement par courrier électronique ou édités en format pdf, de conserver tous les documents relatifs à la prise en charge sous forme numérisée et d'éditer des courriers relatifs à la prise en charge des personnes à risque élevé de cancer. Ces derniers sont automatisés en fonction des champs complétés dans les différents formulaires et sont donc adaptés au suivi spécifique de chaque patient. Par exemple, si une chirurgie prophylactique a été réalisée, l'outil modifie automatiquement le plan personnalisé de suivi pour l'adapter à ce nouvel élément. L'automatisation des courriers représente un gain de temps considérable pour l'utilisateur, sélectionne des items dans des menus déroulants et complète des champs libres avant d'éditer le compte rendu. Une option permettant de marquer les courriers envoyés facilite la tâche de l'utilisateur. Ce logiciel améliore l'efficacité de la prise en charge et, selon les choix personnels des utilisateurs, a la capacité d'éviter tout dossier " papier ». Les fonctions de requête permettent de sélectionner et trier les données contenues dans le logiciel qui peuvent être exportées en texte tabulé, utilisable pour publipostage. De plus, une fonction de requête dédiée permet d'établir des rapports d'activité mensuels et annuels, demandés par les établissements de soins et les tutelles. Cet outil développé par une structure académique est souple et évolutif et permet de répondre aux exigences actuelles et futures de l'oncogénétique.

\footnotetext{
Remerciements : ce travail a été financé grâce à un contrat de collaboration avec la SAS PIGe0n.
}

Déclaration de liens d'intérêts : les auteurs déclarent ne pas avoir de liens d'intérêts.

\section{Références}

[1] Sussner KM, Jandorf L, Valdimarsdottir $H B$ Educational needs about cancer family history and genetic counselling for cancer risk among frontline healthcare clinicians in New York City. Genet Med 2011;13:785-93.

[2] Albada A, Ausems MG, Otten R, Bensing JM, van Dulmen $S$. Use and evaluation of an individually tailored website for counselees prior to breast cancer genetic counselling. J Cancer Educ 2011;26:670-81.

[3] Gammon A, kohlmann W, Burt R. Can we identify the high-risk patients to be screened? A genetic approach. Digestion 2007;76:7-19.

[4] Rolnick SJ, Rahm AK, Jackson JM, Nekhlyudov L, Goddard KA, et al. Barriers in identification and referral to genetic counselling for familial cancer risk: the perspective of genetic service providers. J Genet Couns 2011;20:314-22

[5] Armel SR, Hitchman K, Millar K, Zahavich L, Demsky $R$, et al. The use of family history questionnaires: an examination of genetic risk estimates and genetic testing eligibility in the non-responder population. I Genet Couns 2011;20:355-64.

[6] Aiello-Laws L. Genetic cancer risk assessment. Semin Oncol Nurs 2011;27:13-20.

[7] Décret $n^{0}$ 2000-970 du 23 juin 2000

[8] Décret $n^{0}$ 2013-527 du 20 juin 2013

[9] Oncogénétique en 2014 : consultations, laboratoires et prise en charge. Collection Appui à la décision; 2016:41 [Available from: http://www.e-cancer.fr/Expertises-etpublications/Catalogue-des-publications/
Oncogenetique-en-2014-Consultationslaboratoires-et-prise-en-charge]

[10] PostgresQLFr. Available from: http://www. postgresqlfr.org/.

[11] PHP. Available from: http://www.php.net/.

[12] W3schools HTML tutorial. Available from: http://www.w3schools.com/html/.

[13] W3schools CSS tutorial. Available from: http://www.w3schools.com/css/default. asp.

[14] W3schools JavaScript tutorial. Available from: http://www.w3schools.com/js/default.asp.

[15] http://www.w3schools.com/jquery/ jquery_ajax_intro.asp.

[16] http://www.fpdf.org/.

[17] http://phpmailer.worxware.com/. 